\title{
The eye and the kidney: twin targets in diabetes
}

\author{
T. Ravi Raju ${ }^{1,2}$ - N. V. Madhavi ${ }^{3}$ G. R. Sridhar ${ }^{4}$
}

Published online: 19 December 2015

(C) Research Society for Study of Diabetes in India 2015

Diabetes mellitus is both defined by and is a dreaded condition because of microvascular involvement of the eye and the kidneys. The level of glucose at which retinopathy occurs forms the basis for diagnosing diabetes. Despite non-invasive methods being available for its diagnosis, retinopathy is often underdiagnosed and untreated. Eventually it leads to visual loss often in working-age populations.

There appears to be ethnic variation in susceptibility to diabetic retinopathy (DR), with prevalence of DR, sightthreatening DR and macular edema being higher in people from South Asia, Africa, Latin America and indigenous tribal populations [1]. The variations are ascribed to differential susceptibility to known risk factors, as well as differences in lifestyle, access to health care and genetic and epigenetic phenomenon. A number of prevalence studies were reported from different parts of India. In a hospital-based report from $\operatorname{Kashmir}(n, 500$ with diabetes), DR was identified in $27 \%$ $(n, 135)$ [2]. Increasing age was a risk factor, and milder forms accounted for the most cases, suggesting that early screening and treatment is necessary [3].

Prevalence studies from rural India are fewer [4]. In a population-based Central India Eye and Medical Study involving more than 4500 subjects, DR was diagnosed by fundus photography utilizing criteria of Early Treatment of

G. R. Sridhar

sridharvizag@gmail.com

1 NTR University of Health Sciences, Vijayawada, India

2 Department of Nephrology, Andhra Medical College, Visakhapatnam, India

3 Dr GM Narayanaswamy Medical Centre, Visakhapatnam, India

4 Endocrine and Diabetes Centre, 15-12-15 Krishnanagar, Visakhapatnam 530002, India
Diabetic Retinopathy Study. DR was identified in 15 subjects $(0.33 \%)$ of the entire cohort. In subjects with diabetes, DR was present in $9.6 \%$ [5]. Risk factors were increasing age and higher glucose concentrations. All eyes showed only nonsevere forms of DR. There was no significant association with other conventional risk factors or with ocular parameters. In the SN-DREAMS III report no 2 (Sankara Nethralaya Diabetic Retinopathy Epidemiology and Molecular Genetic Study III), population-based cross-sectional screening was carried out in a rural population of south India $(n, 13,079)$ [6]. DR was identified using $45^{\circ}$ four-field stereoscopic digital photography, along with $30^{\circ}$ seven-field stereo digital pairs in those with DR. Among those with diabetes, DR was identified in $10.3 \%$, with greater risk in men, insulin users, those with longer duration of diabetes, systolic hypertension and poor glycemic control [6].

In urban Chennai, the CURES Eye Study I, a populationbased screening for diabetes, was carried out in adults aged 20 years and above $(n, 26,001)$. Among 1382 known subjects with diabetes who agreed to undergo four-field stereo colour photography, and 354 newly diagnosed subjects with diabetes, DR was present in $17.6 \%$ [7]. The prevalence of DR was higher in men and those with proteinuria.

Another population-based study in South Kerala assessed the prevalence of diabetes and DR in a community-based screening programme. One hundred and sixty camps were held in five southern districts of Kerala (Project Trinetra) [8].

In a pilot hospital-based screening for DR from western India $(n, 168)$ using ETDRS classification, prevalence of DR was $33.9 \%$; non-proliferative DR was $25.5 \%$ and proliferative DR $8.33 \%$ [9].

The current issue of the Journal reports on the risk factors for DR in sub-Saharan Africa, prevalence in a rural south Indian population, and on biomarkers for DR and screening methods. 
Photography of the retinal vessels has progressed from analog [10] to digital. Despite the convenience of non-mydriatic camera to screen for DR, multiple field stereo photography is necessary for precise classification. Genetic and epigenetic factors were linked to the development of DR. Microvascular dysfunction results from involvement of breakdown of blood retinal barrier, capillary basement thickening involving different cellular components of the retina including the retinal neuronal and glial dysfunction. Pathogenic pathways include altered retinal blood flow, inflammatory pathways and growth factors such as vascular endothelial growth factor (VEGF) [11]; the latter forms the target for treatment of DR by the use of drug modulators.

Diabetic nephropathy (DN) is the other major diabetic microvascular complication accounting for significant morbidity and mortality. Metabolic and genetic factors contribute to its pathogenesis. This issue carries reports on DN relating to risk factors, early recognition, biomarkers and intervention. At the outset, one should bear in mind that non-diabetic renal disease could contribute to renal failure in diabetes mellitus. Clinical situation simplying non-diabetic renal disease includes shorter duration of diabetes, microscopic hematuria and active urinary sediment in the absence of retinopathy [12]. An Indian study showed a poor correlation between DN and DR in type 2 diabetes mellitus and suggested that renal biopsy is useful in staging the lesions in subjects with diabetes having renal dysfunction [13]. A similar discordance between DR and DN in type 2 diabetes was reported, advocating the requirement of renal biopsy for a precise renal pathological diagnosis in these situations [14].

A number of biomarkers were studied to identify early stages of renal involvement in diabetes, which may pre-date the clinical diagnosis of diabetes. Markers of tubular damage such as urine neutrophil gelatinase-associated lipocalin and cystan $\mathrm{C}$ are potential biomarkers [15]. Other putative biomarkers include serum prolidase activity, which is associated with oxidative stress, also contributing to diabetic nephropathy; oxidant stress is reported as total oxidant stress, total antioxidative status and oxidative stress index [16]. Newer biomarkers employing proteomics and emerging omics technologies are likely to improve the capability to identify nephropathy at an early stage and allow aggressive interventions to prevent further progress [17]. Whereas urinary miRNAs could serve as biomarkers in diabetic complications, a recent study reported that among five detectable TGF- $\beta 1$-regulated miRNAs, only circulating levels of let-7b-5p and miR-21-5p were associated with risk of end-stage renal disease [18]. However, one must bear in mind that circulating miRNA level may not correlate with tissue levels [19].

Much focus has been directed towards the role of genetic factors contributing to DR, in view of the availability of sequencing techniques. It helps in serving as biomarkers, and in providing information into pathogenesis, and opening up avenues for new drug targets. Studies targeted gene variants of inflammatory cytokine genes (interleukins, tumour necrosis factor), extracellular matrix components (collagen, laminins, matrix metalloproteinase 9), renal function (angiotensinogen and angiotensin II receptors), endothelial function and oxidative stress (nitric oxide synthase 3 , catalase, superoxide dismutase 2), and glucose and lipid metabolism (adiponectin, Apolipoprotein E, aldose reductase, glucose transporter 1, peroxisome proliferator-activated receptor gamma 2) [20].

There have been a number of Indian studies on candidategene approach to DR. Vascular endothelial growth factor (VEGF), a potent cytokine, has a role in the pathogenesis of diabetic microvascular disease, including DN. A pilot crosssectional study $(n, 40)$ from a North Indian population showed that DD genotype and D allele in I/D polymorphism at -2549 position of VEGF gene increased the susceptibility to DN [21]. Polymorphisms of osteopontin promoter were reported to be a genetic risk factor for DN. In a case-control study, three functional promoter gene polymorphisms and their haplotypes were studied in relation to risk of DN (C-443T, delG-156G, G-66T) [22]. The results suggested an association between the gene promoter polymorphisms and their haplotypes with risk of DN in subjects with T2DM. Transcription factor 7-like 2 (TCF7L2) gene is associated with DN. In a south Indian population, polymorphisms of the gene were studied in relation to DN [23]. The rs12255372 polymorphism in the TCF7L2 gene is associated with DN but operates through diabetes mellitus.

A larger sample from Punjab and from Jammu and $\operatorname{Kashmir}(n, 1313)$ was genotyped for TGF- $\beta 1$ (rs 1800470 and rs1800469). The CC genotype of rs1800470 increased the risk of end-stage renal disease by 3.1-4.5-fold [24]. TNF promoter polymorphism, associated with chronic low-grade inflammation, was studied in relation to susceptibility to $\mathrm{DN}$. It was shown that $863 \mathrm{C} / \mathrm{A}$ polymorphism might be protective against $\mathrm{DN}$ whereas $-1031 \mathrm{~T} / \mathrm{C}$ may increase the risk of $\mathrm{DN}[25]$.

Data on ACE2 is available from different areas of India. Angiotensin 1 receptor and angiotensin 2 receptors regulate NF-kB and ACE2, and provide a potential for identifying drug targets [26]. ACE (I/D) gene polymorphisms were reported to predispose to DN from Kutch region in western India. About 150 each of Ahir and Rabri ethnic groups with diabetes of more than 10 years were studied. Variant at ACE locus as $\mathrm{D} / \mathrm{D}$ variant in intron 16 contributed to increased risk but not severity of DN [27]. Genes involved in oxidative stress pathway appear to predispose to DN [28, 29]. Replication studies for TGF $\beta 1$ gene and for SNPs in RAGE and GFPT2 in India were obtained [30, 31].

Eventually, most people with end-stage diabetic renal disease require long-term dialysis or renal transplant. While the technology for the latter is available, lack of adequate donors is a bottleneck. Monitoring the renal status for early signs of rejection is an important aspect in the post-transplant stage. 
Methods for non-invasive detection of renal allograft inflammation by measuring mRNA for IP-10 and CXCR3 in urine were shown to be a potential biomarker of acute rejection [32].

Current approach to managing DR is limited to the later stages of the condition and does not address the early stages where reversing the change can be considered. Anti-VEGF treatment is an advance, but other mechanisms can be targeted involving the retinal involvement of glia, neurons and vessels [11]. Emerging treatments in the pipeline include modifying expression of antioxidant genes, the kallekrein inhibitors and NOX-2 inhibitors. Stem cell reparative processes are also being studied in the treatment of DR [11].

Approach to DN is also multifactorial, controlling glucose levels, blood pressure lipids and protein intake in the diet. Further understanding of the pathogenic mechanisms shows promise of newer drug targets against pathways upregulated by elevated glucose levels as well as others leading to progression of DN [33]. Recent GWAS investigations show promise of identifying novel loci influencing albuminuria in diabetes [34].

The mix of articles in this issue reflects the trend for greater focus of genetic studies in DN compared to DR.

\section{References}

1. Sivaprasad S, Gupta B, Nwaobi RC, Evans J. Prevalence of diabetic retinopathy in various ethnic groups: a worldwide perspective. Surv Ophthalmol. 2012;57:347-70.

2. Qureshi T, Abdullah N, Shagufta. Prevalence of diabetic retinopathy in Kashmir, India - a hospital based study. Global J Med Public Health. 2013;2:1. www.gjmedph.com.

3. Rema M, Pradeepa R. Diabetic retinopathy: an Indian perspective. Indian J Med Res. 2007;125:297-310.

4. Mohan V, Pradeepa R. Risk factors for diabetic retinopathy in rural India. J Postgrad Med. 2009;55:89-90.

5. Jonas JB, Nangia V, Khare A, et al. Prevalence and associated factors of diabetic retinopathy in rural Central India. Diabetes Care. 2013;36:e69.

6. Raman R, Ganesan S, Pal SS, Kulothungan V, Sharma T. Prevalence and risk factors for diabetic retinopathy in rural India. Sankara Nethralaya Diabetic Retinopathy Epidemiology and Molecular Genetic Study III (SN-DREAMS III), report no 2. BMJ Open Diab Res Care. 2014;2:000005. doi:10.1136/bmjdrc2013-000005.

7. Rema M, Premkumar S, Anitha B, Deepa R, Pradeepa R, Mohan V. Prevalence of diabetic retinopathy in urban India: the Chennai Urban Rural Epidemiology Study (CURES) Eye Study, I. Invest Ophthalmol Vis Sci. 2005;46:2328-33.

8. Soman M, Nair U, Bhilal S, Mathew R, Gafoor F, Nair KGR. Population based assessment of diabetes and diabetic retinopathy in South Kerala-Project Trinetra: an interim report. Kerala J Ophthalmol. 2009;1:36-41.

9. Ramavat PR, Ramavat MR, Ghugare BW, Vaishnav RG, Joshi MU. Prevalence of diabetic retinopathy in western Indian type 2 diabetic population: a hospital-based cross-sectional study. J Clin Diagn Res [serial online]. 2013;7:1387-90.
10. Sridhar GR, Satish K, Ahuja MMS. Non mydriatic retinal color photography in young Indian diabetics. Ann Ophthalmol. 1993;25:187-90.

11. Stitt AW, Lois N, Medina RJ, Adamson P, Curtis TM. Advances in our understanding of diabetic retinopathy. Clin Sci. 2013;125:1-17.

12. Wilfred DC, Mysorekar VV, Venkataramana RS, Eshwarappa M, Subramanyan R. Non-diabetic renal disease in type 2 diabetes mellitus patients: a clinicopathological study. J Lab Physicians. 2013;5:94-9.

13. Sahay M, Mahankali RK, Ismail K, Vali PS, Sahay RK, Swarnalata. Renal histology in diabetic nephropathy: a novel perspective. Indian J Nephrol. 2014;24:226-31.

14. Prakash J, Gupta T, Prakash S, Bhushan P, Usha SM, Singh SP. Non-diabetic renal disease in type 2 diabetes mellitus: study of renal-retinal relationship. Indian J Nephrol. 2015;25:222-8.

15. Garg V, Kumar M, Mahapatra HS, Chitkara A, Gadpayle AK, Sekhar V. Novel urinary biomarkers in pre-diabetic nephropathy. Clin Exp Nephrol 2015

16. Verma AK, Subhash Chandra, Singh RG, Singh TB, Srivastava S, Srivastava R. Serum prolidase activity and oxidative stress in diabetic nephropathy and end stage renal disease: a correlative study with glucose and creatinine. Biochem Res International 2014. (http://dx.doi.org/10.1155/2014/291458)

17. Rao PV, Lu X, Standle M, et al. Proteomic identification of urinary biomarkers of diabetic nephropathy. Diabetes Care. 2007;30(3): 629-37.

18. Pezzolesi MG, Satake E, McDonald KP, Major M, Smiles AM, Krolewski AS. Circulating TGF- $\beta 1$-regulated miRNAs and the risk of rapid progression to ESRD in type 1 diabetes. Diabetes. 2015;64: 3285-93.

19. Badal SS, Danesh FR. Diabetic nephropathy: emerging biomarkers for risk assessment. Diabetes. 2015;64:3063-5.

20. Rizvi S, Raza ST, Mahdi F. Association of genetic variants with diabetic nephropathy. World J Diab. 2014;5:809-16.

21. Amle D, Mir R, Khaneja A, Agarwal S, Ahlawat R, Ray PC, et al. Association of $18 \mathrm{bp}$ insertion/deletion polymorphism, at -2549 position of VEGF gene, with diabetic nephropathy in type 2 diabetes mellitus patients of North Indian population. J Diab Metab Dis. 2015;14:19. doi:10.1186/s40200-0144-3.

22. Cheema BS, Iyengar S, Sharma R, Kohli HS, Bhansali A, Khullar M. Association between osteopontin promoter gene polymorphisms and haplotypes with risk of diabetic nephropathy. J Clin Med. 2015;4:1281-92.

23. Bodhini D, Chidambaram M, Liju S et al. Association of TCF7L2 polymorphism with diabetic nephropathy in the south Indian population. Ann Human Genet 2015. doi:10.1111/ahg.12122

24. Raina P, Sikka R, Kaur R, et al. Association of transforming growth factor beta-1 (TGF- $\beta 1$ ) genetic variation with type 2 diabetes and end stage renal disease in two large population samples from north India. OMICS. 2015;19:306-17.

25. Gupta S, Mehndiratta M, Kalra S, Kalra OP, Shukla R, Gambhir JK. Association of tumour necrosis factor (TNF) promoter polymorphisms with plasma TNF-a levels and susceptibility to diabetic nephropathy in North Indian population. J Diab Complications. 2015;29:338-42.

26. Pandey A, Goru SK, Kadakol A, Malek V, Gaikwad AB. Differential regulation of angiotensin converting enzyme 2 and nuclear factor-kB by angiotensin II receptor subtypes in type 2 diabetic kidney. Biochimie 2015. doi:10.1016/j.biochi.2015.08. 005

27. Parchwani DN, Palandurkar KM, Hemachandan Kumar D, Patel DJ. Genetic predisposition to diabetic nephropathy: evidence for a role of $\mathrm{ACE}(\mathrm{I} / \mathrm{D})$ gene polymorphism in type 2 diabetic population from Kutch region. Indian J Clin Biochem. 2015;30:43-54. 
28. Dabhi B, Mistry KN. Oxidative stress and its association with TNFa-308G/C and IL-1a-889 C/T gene polymorphisms in patients with diabetes and diabetic nephropathy. Gene. 2015;562:197-202.

29. Narne P, Ponnaluri KC, Siraj M, Ishaq M. Polymorphisms in oxidative stress pathway genes and risk of diabetic nephropathy in south Indian type 2 diabetic patients. Nephrol (Carlton). 2014;19:623-9.

30. Prasad P, Tiwari AK, Prasanna Kumar KM, Ammini AC, Arvind G, Rajeev G, et al. Association of TGF $\beta 1, T N F \alpha$, CCR2 and CCR5 gene polymorphisms in type-2 diabetes and renal insufficiency among Asian Indians. BMC Med Genet. 2007;8:20. doi:10.1186/ 1471-2350-8-20.

31. Prasad P, Tiwari AK, Prasanna Kumar KM, Ammini AC, Arvind G, Rajeev G, et al. Association analysis of ADPRT1, AKR1B1,
RAGE, GFPT2 and PAI-1 gene polymorphisms with chronic renal insufficiency among Asian Indians with type-2 diabetes. BMC Med Genet. 2010;11:52. doi:10.1186/1471-2350-11-52.

32. Ravi Raju T, Muthukumar T, Dadhania D, et al. Noninvasive detection of renal allograft inflammation by measurements of mRNA for IP-10 and CXCR3 in urine. Kidney Int. 2004;65:2390-7.

33. Ahmad J. Management of diabetic nephropathy: recent progress and future perspective. Diabetes Metab Syndr 2015. doi: 10.1016/ j.dsx.2015.02.008

34. Teumer A, Tin A, Sorice R et al. Genome-wide association studies identify genetic loci associated with albuminuria in diabetes. Diabetes 2015 